\title{
Determinantes clínicos com influência no prognóstico da discectomia lombar: análise multifatorial
}

\author{
Clinical determinants' influence on lumbar discectomy prognosis: \\ multi-factor analysis
}

Determinantes clínicos que influyen en el pronóstico de la disectomía lumbar: análisis multifactorial

\author{
Paulo Jorge Da Rocha Carvalho' \\ Paulo Jorge Correia De Sá ${ }^{2}$ \\ Rolando Manuel Bezerra Freitas ${ }^{3}$ \\ João Pedro Maia Gonçalves ${ }^{4}$ \\ Sara Lemos Costa Lobo Macedo ${ }^{5}$ \\ José De Morais Neves ${ }^{6}$
}

\section{RESUMO}

A seleção e o agendamento temporal inadequados dos pacientes permanecem as causas mais importantes de insucesso da cirurgia discal lombar. Objetivo: Este trabalho pretende avaliar os resultados clínicos e os factores preditivos que influenciam o prognóstico dos pacientes submetidos à discectomia lombar através de um modelo analítico multifatorial. Métodos: Apresentamos uma série prospectiva de 68 pacientes com hérnias discais lombares que foram submetidos à discectomia entre 2003 e 2007 . O segui-

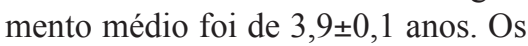
resultados clínicos foram avaliados utilizando o escore JOA (Japonese Orthopaedic Association Assessment Scoring System) (JOA=29). Resultados: Resultados pós-operatórios bom e excelente foram obtidos em $75 \%$ dos casos. Pacientes do sexo masculino e a preponderância de sintomas subjectivos sobre sintomas clínicos estiveram associados com os melho-

\section{ABSTRACT}

Inappropriate patient selection and timing for lumbar disc surgery are still the the major causes of failures. Objective: We evaluated the clinical outcome and predictive factors that influence the prognosis of patients submitted to lumbar discectomy using a multivariate analysis model. Methods: We report a prospective series of 68 patients with herniated lumbar disc who underwent limited discectomy during 2003 to 2007. The average follow-up period was $3.9 \pm 0.1$ years. Clinical findings were evaluated using Japonese Orthopaedic Association Assessment Scoring System $(J O A=29)$. Results: Excellent and good postoperative improvement rates were obtained in $75 \%$ of the patients. Male gender and preponderance of subjective symptoms over clinical signs were associated with better clinical outcomes and faster return to work. Waiting time for surgery had no
\end{abstract}

\section{RESUMEN}

La selección y la agenda temporal inadecuada de los enfermos permanecen como las causas más importantes de la falta de éxito en la cirugía discal lumbar. Objetivo: este trabajo pretende evaluar los resultados clínicos y los factores predictivos que influyen en el pronóstico de los pacientes sometidos a la discectomía lumbar por medio de un modelo analítico multifatorial. Métodos: se presenta una serie prospectiva de 68 pacientes con hernias discales lumbares, los cuales fueron sometidos a la discectomía entre 2003 y 2007. El seguimiento

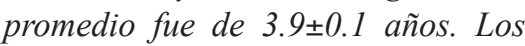
resultados clínicos fueron evaluados utilizando el JOA (Japonese Orthopaedic Association Assessment Scoring System) score $(J O A=29)$. Resultados: los resultados postoperatorios buenos y excelentes fueron obtenidos en $75 \%$ de los pacientes. Enfermos del sexo masculino y la preponderancia de síntomas subjetivos sobre sin-

\footnotetext{
Trabalho realizado no Serviço de Ortopedia e Traumatologia, Centro Hospitalar VN Gaia, Espinho EPE, Vila Nova de Gaia, Portugal.

'MD, Interno Complementar de Ortopedia e Traumatologia do Centro Hospitalar de Vila Nova de Gaia, Espinho EPE, Portugal

${ }^{2} \mathrm{MD}$, PhD, Professor de Farmacologia da Faculdade Ciências Médicas Abel Salazar; Hospital de Santo António, Portugal.

${ }^{3} \mathrm{MD}$, Chefe de Serviço de Ortopedia e Traumatologia do Centro Hospitalar de Vila Nova de Gaia, Espinho EPE, Portugal.

${ }^{4} \mathrm{MD}$, Assistente de Ortopedia e Traumatologia do Centro Hospitalar de Vila Nova de Gaia, Espinho EPE, Portugal.

${ }^{5} \mathrm{MD}$, Interna Complementar de Ortopedia e Traumatologia do Centro Hospitalar de Vila Nova de Gaia, Espinho EPE, Portugal.

${ }^{6} \mathrm{MD}$, Director de Serviço de Ortopedia e Traumatologia do Centro Hospitalar de Vila Nova de Gaia, Espinho EPE, Portugal.
} 
res resultados clínicos e com o retorno mais precoce à actividade laboral. O tempo de espera pela cirurgia não teve influência no resultado final. A necessidade de cirurgia foi questionada na ausência de défices neurológicos significativos (JOA para resultados clínicos $\geq 3 / 6$ ). Um valor JOA pré-operatório $\geq 15$ (debilidade inicial baixa) está correlacionado com piores resultados clínicos pós-operatórios. Conclusões: $\mathrm{O}$ escore JOA é de fácil aplicação para clínicos e pacientes, uniformiza dados subjectivos e apresenta valor preditivo para os resultados dos pacientes submetidos à discectomia lombar. consistent effect on the outcome. The need for immediate surgery was questionable in the absence of significant neurological disturbance (JOA for clinical signs $\geq 3 / 6$ ). JOA scoring had predictive value for preoperative assessment for lumbar disc surgery, i.e. preoperative JOA $\geq 15$ (low initial disability) correlated with poor surgery outcome. Conclusions: JOA scoring is easily performed for patients and clinicians, standardizes subjective data and presents high predictive value of patients' outcome after lumbar discectomy

KEYWORDS Intervertebral disk displacement/surgery; Low back pain; Lumbar vertebrae/ pathology tomas clínicos estuvieron asociados con los mejores resultados clínicos y con el regreso más precoz a la actividad laboral. El tiempo de espera para la cirugía no influyó en el resultado final. La necesidad de cirugía fue cuestionada en la ausencia de déficits neurológicos significativos (JOA para resultados clínicos $\geq 3 / 6$ ). Un valor $J O A$ preoperatorio $\geq 15$ (debilidad inicial baja) está correlacionado con los peores resultados clínicos postoperatorios. Conclusiones: el JOA score se presenta de fácil aplicación para clínicos y enfermos, uniformiza datos subjetivos y presenta un valor predictivo para los resultados de los pacientes sometidos a la discectomía lumbar.

DESCRIPTORES: Desplazamiento del disco intervertebral/ cirugía; Dolor de la región lumbar; Vértebras lumbares/ patología

\section{INTRODUÇÃO}

Estima-se que entre 60 e $80 \%$ da população adulta tenha queixas de lombalgia baixa, 2 e $5 \%$ anualmente. A vasta maioria, provavelmente maior do que $90 \%$, recupera-se das queixas lombares sem necessidade de tratamento cirúrgico. Apenas 2 a 3\% da população com lombalgia tem hérnia discal lombar e cerca de $1 \%$ apresenta compressão de uma raiz ${ }^{1}$. Na maioria dos casos, as queixas álgicas melhoram espontaneamente com tratamento conservador em 4 a 6 semanas, com períodos de repouso ( $\leq 5$ dias), com a utilização de anti-inflamatórios não-esteroides, exercícios aeróbicos e medidas higieno-dietéticas (incluem alterações de actividade física $)^{2}$. Quando as queixas álgicas persistem (cerca de $2 \%$ dos casos) ou a radiculopatia permanece (10 a $25 \%$ dos casos), afigura-se como necessário o tratamento cirúrgico para menos de $0,5 \%$ da população ${ }^{3}$.

O papel da cirurgia no tratamento das hérnias discais lombares sintomáticas tem sido matéria de debate na literatura. Alguns autores defendem que a cirurgia tem um papel puramente paliativo, enquanto outros demonstram que os pacientes submetidos à discectomia lombar apresentam melhores resultados durante o primeiro ano e que se mantêm com o tempo ${ }^{4}$. Contudo, os maiores benefícios do tratamento cirúrgico, nas hérnias discais lombares sintomáticas, aparentam ser um alívio mais rápido da dor relativa à compressão radicular e uma aceleração da melhoria das alterações neurológicas em relação ao tratamento conservador.
Os exames pré-operatórios podem apresentar dificuldades de interpretação ou resultados falso-negativos/positivos. Sabe-se que 35\% dos indivíduos assintomáticos apresentam uma alteração discal na mielografia, tomografia computadorizada (TC) ou ressonância magnética (RMN), sendo que, em $20 \%$ dos casos com idade $\leq 60$ anos, evidencia-se herniação discal ${ }^{5}$. Complementando os exames com a avaliação de sinais neurológicos ou factores psicológicos, verifica-se melhor correlação com os achados cirúrgicos, e melhoram significativamente as indicações da discectomia ${ }^{6}$. A electromiografia (EMG) apresenta pouca utilidade a menos que seja detectada uma neuropatia periférica. Por outro lado, estudos prospectivos provaram que os resultados iniciais da EMG não têm significado prognóstico na determinação do resultado da cirurgia discal lombar aos cinco anos de seguimento ${ }^{7}$.

A selecção e o agendamento temporal inadequados permanecem as causas mais importantes de insucesso da cirurgia discal lombar. Os estudos de avaliação de resultados da cirurgia discal lombar documentam uma taxa de sucesso que varia entre 49 e $90 \%$, quando usados critérios de avaliação adequados ${ }^{2,8-10}$. Vários autores concluíram que a utilização de um sistema de avaliação objectivo melhora os resultados clínicos após discectomia selectiva ${ }^{11}$. Embora os factores psicossociológicos sejam reconhecidamente factores preditivos negativos dos resultados da cirurgia discal lombar ${ }^{12}$, a eficácia dos testes psicológicos requer o preenchimento de 
longos questionários e/ou a realização de entrevistas exaustivas, que são dificilmente exequíveis na prática clínica diária. Nesta perspectiva, o nosso objectivo foi a avaliação, através de um modelo analítico multifatorial facilmente aplicável, dos resultados clínicos e dos factores preditivos que influenciam o prognóstico a longo termo ( $\geq 3$ anos) dos pacientes submetidos à discectomia lombar seletiva.

\section{MÉTODOS}

\section{Pacientes e procedimentos cirúrgicos}

Oitenta e oito pacientes com hérnias discais lombares (confirmadas por TC ou RMN), cujo tratamento conservador falhou, foram submetidos, pela primeira vez, à discectomia lombar selectiva, no Serviço de Ortopedia e Traumatologia do Centro Hospitalar de Vila Nova de Gaia, entre 2003 e 2007.

Cinquenta e quatro pacientes eram do sexo masculino e 34 do sexo feminino, com média de idade, na altura da cirurgia, de 39 \pm 5 anos (idades entre 19-59 anos).

Vinte $(22,7 \%)$ dos 88 pacientes não foram possíveis de contactar, resultando num valor total de doentes semelhante ao apresentado em outros estudos ${ }^{5,13}$, suficiente para avaliação com objectivo de análise estatística. Os restantes 68 doentes foram incluídos no presente estudo.

O tratamento conservador consistiu em breves períodos de repouso ( $\leq 5$ dias), anti-inflamatórios nãoesteroides, fisioterapia e medidas dietéticas. Com o insucesso deste tipo de tratamento ao fim de oito semanas, os pacientes foram inscritos para tratamento cirúrgico.

Doentes com cirurgias lombares anteriores, doenças sistémicas ou outros factores de comorbilidade associados, fumadores ou toxicodependentes não foram incluídos nesta série.

O procedimento cirúrgico utilizado foi a microdiscectomia com laminotomia parcial. A intervenção foi realizada por uma equipa de três cirurgiões da coluna experientes.
O tempo de espera para a cirurgia variou de 3 a 15 meses. L4-L5 foi o espaço envolvido em $49 \%$ dos pacientes, L5-S1 em 47\% e L3-L4 em 1\%. Em 3\% dos pacientes, verificou-se envolvimento de mais do que 1 nível.

\section{Seguimento}

Um investigador independente avaliou os pacientes prospectivamente, depois de estabelecida a indicação da discectomia. O período de seguimento médio foi de 3,9 90,1 anos (variação de 3 a 5 anos). Quatorze pacientes foram seguidos durante 5 anos, 24 durante 4 anos e 30 durante 3 anos (Tabela 1).

Os dados clínicos pré-operatórios e os resultados foram avaliados segundo o Japanese Orthopaedic Association Assessment Scoring System (escore JOA - Tabela 2) ${ }^{5}$, que inclui sintomas subjectivos ( 9 pontos), sinais clínicos (6 pontos) e restrição das actividades da vida diária (RAQ, 14 pontos). A avaliação da melhoria pós-operatória (IR) resultou do quociente: [(escore JOA pós-op - escore JOA pré-op)/(29 - escore JOA pré-op)] x $100 \%$. O resultado foi classificado como excelente quando IR $\geq 80 \%$, bom quando IR entre 65 e $80 \%$, suficiente quando IR entre 50 e $65 \%$ e insuficiente quando IR $\leq 50 \%$.

\section{Análise multifatorial e análise estatística}

Os dados da análise multifatorial incluíram a idade do paciente na altura da cirurgia, sexo, peso, tempo de espera para o procedimento, complicações, retorno à actividade laboral e taxa de melhoria clínica pós-operatória (IR). Foram calculadas as frequências em valor absoluto e percentual para as variáveis contínuas, assim como as médias e os desvios padrões das variáveis discretas.

Os dados foram apresentados como \pm S.E.M. para $n$ observações. O significado estatístico das diferenças foi avaliado pela análise da variância (ANOVA) seguida pelo teste $t$ de Dunnett modificado. O valor de $\mathrm{p}<0,05$ foi considerado significativo.

TABELA 1 - Análise multifatorial dos resultados

\begin{tabular}{|c|c|c|c|c|c|}
\hline & $\begin{array}{c}\text { Excelente } \\
(n=36)\end{array}$ & $\begin{array}{c}\text { Bom } \\
(n=16)\end{array}$ & $\begin{array}{c}\text { Suficiente } \\
(n=10)\end{array}$ & $\begin{array}{c}\text { Insuficiente } \\
(n=6)\end{array}$ & Valor de $p$ \\
\hline Melhoria (\%) & $90,1 \pm 7,9$ & $68,6 \pm 2,7$ & $58,3 \pm 3,0$ & $10,5 \pm 4,0$ & \\
\hline Idade na data da cirurgia & $39,4 \pm 9,1$ & $39,1 \pm 10,4$ & $40,1 \pm 9,5$ & $40,5 \pm 12,8$ & n.s. \\
\hline $\operatorname{Sexo}\left(0^{\pi} /\right.$ 우 $)$ & 6,7 & 0,7 & 0,8 & 0,3 & $<0,05$ \\
\hline \multicolumn{6}{|c|}{ Tempo de espera para a cirurgia (\%) } \\
\hline $0-3$ meses $-38 \%$ & 39 & 45 & 42 & - & n.s. \\
\hline $3-6$ meses - $29 \%$ & 22 & 18 & 29 & 100 & n.s. \\
\hline $6-12$ meses - $24 \%$ & 35 & 27 & - & - & n.s. \\
\hline$>12$ meses - $9 \%$ & 4 & 10 & 29 & - & $<0,05$ \\
\hline Complicações (\%) & 17 & 18 & 42 & 50 & $<0,05$ \\
\hline Retorno ao trabalho (\%) & 96 & 73 & 64 & 25 & $<0,05$ \\
\hline \multicolumn{6}{|l|}{ Seguimento (\%) } \\
\hline 5 anos - $34 \%$ & 22 & 27 & - & 25 & n.s. \\
\hline 4 anos - $44 \%$ & 39 & 27 & 43 & 25 & n.s. \\
\hline 3 anos - $52 \%$ & 39 & 46 & 57 & 50 & n.s. \\
\hline
\end{tabular}

n.s.: não significativo. 


\section{RESULTADOS}

\section{Resultados pré e pós-operatórios}

O valor médio pré-operatório do JOA score foi de 14,8 $\pm 4,2$ para os 88 pacietnes inicialmente incluídos no estudo. Este valor não foi significativamente diferente $(\mathrm{p}>0,05)$ do valor do escore JOA dos 68 pacientes restantes que foram contactados $(14,5 \pm 4,3)$, indicando portanto que os dois grupos eram homogéneos. Por outro lado, após análise dos registros pós-operatórios dos pacientes (que não foram possíveis de contactar para a entrevista de seguimento), verificou-se que os parâmetros variáveis, como a idade na data da cirurgia $(38 \pm 5$ anos), quociente entre sexos $(1,5)$ e complicações associadas (12\%), não foram significativamente diferentes do grupo entrevistado. Praticamente todos os participantes apresentavam lombalgia com radiculopatia associada $(58,85 \%)$ como sintoma inicial; 9 pacientes $(13 \%)$ apresentavam somente dor radicular no membro inferior envolvido e 1 paciente $(2 \%)$ apresentava unicamente lombalgia.

A taxa média de melhoria pós-operatória (IR) após discectomia lombar selectiva foi de $73 \%$, correspondendo a um resultado global médio bom (Figura 1); O valor JOA pós-operatório $(25,4 \pm 2,8, n=68)$ foi significativamente $(\mathrm{p}<0,05)$ superior ao valor pré-operatório $(14,5 \pm 4.3$, $n=68$ ). Como evidenciado na figura 1 , a taxa de melhoria geral percentual, quando comparados os escores JOA pré e pós-operatório, foi significativamente superior para sintomas subjectivos, principalmente lombalgia e dor radicular, em relação aos sinais objectivos, nomeadamente alterações sensitivas e motoras, que apresentaram apenas melhorias discretas no pós-operatório. A melhoria das funções sensoriais das fibras nervosas pode demorar mais de um ano, e depende da gravidade da lesão e do diâmetro das fibras nervosas ${ }^{11}$. Como já foi enfatizado por outros autores $^{14}$, a melhoria pós-operatória do teste de Lassègue apresenta uma forte correlação com a redução da dor radicular e a melhoria da capacidade da marcha, sugerindo um bom resultado cirúrgico. Apresentaram também francas melhorias na capacidade de realização de actividades quotidianas restritas antes da cirurgia.

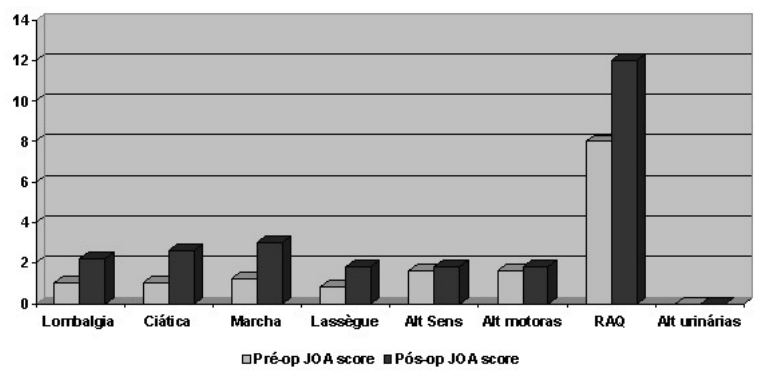

Figura 1

Comparação entre os valores JOA pré e pós-operatório em relação aos sintomas subjectivos, sinais clínicos, restrições das actividades quotidianas (RAQ) e alterações urinárias. Valores

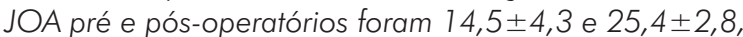
respectivamente $(n=68)$
Em relação à taxa IR pós-operatória, $51 \%$ dos pacientes apresentaram um resultado excelente ( $\mathrm{IR}=90,1 \pm 7,9 \%$, $n=36), 24 \%$ bom (IR=68,6 $\pm 2,7 \%, n=16), 16 \%$ suficiente $(\mathrm{IR}=58,3 \pm 3,0 \%, n=10)$ e $9 \%$ insuficiente $(\mathrm{IR}=10,5 \pm 4,0 \%$, $n=6)$ (Tabela 1). O JOA score apresenta valor preditivo para a decisão pré-operatória para cirurgia discal lombar, uma vez que os resultados insuficientes foram significativamente correlacionados com baixa debilidade pré-operatória, isto é, escore JOA pré-operatório $\geq 15(19,3 \pm 2,7)$ (Figura 2).

\section{Análise multifatorial}

A Tabela 1 sumariza a análise multifatorial dos grupos no fim do período de seguimento. Para identificar factores preditivos relevantes, a quantidade de variáveis relativas à anamnese, assim como ao exame clínico, foram descritas e as diferenças testadas entre os subgrupos. Não foram encontradas diferenças significativas percentuais nos grupos com seguimento de 3, 4 e 5 anos em relação à população total (Tabela 1). Adicionalmente, não houve diferenças significativas nas idades médias na data da cirurgia nestes subgrupos. Os resultados indicaram que o sexo masculino esteve associado a melhores resultados clínicos e a regresso mais célere à actividade laboral. Resultados excelentes estiveram associados a um quociente $\widehat{\delta} / \propto$ de 6.7 , o qual foi significativamente $(\mathrm{p}<0,05)$ superior em relação ao quociente $\widehat{\delta} / q$ da população total (1.6).

Outro parâmetro introduzido na análise multifatorial foi o tempo de espera para a cirurgia (Tabela 1); 67\% dos doentes foram operados nos primeiros seis meses após a indicação para cirurgia. $\mathrm{O}$ atraso para a cirurgia esteve inversamente correlacionado com a intensidade dos sinais clínicos pré-operatórios, isto é, a cirurgia foi mais atrasada nos pacientes com menores défices neurológicos inicialmente (sinais clínicos pré-operatórios $\geq 3$ ). Não foi encontrada correlação significativa entre a debilidade subjectiva

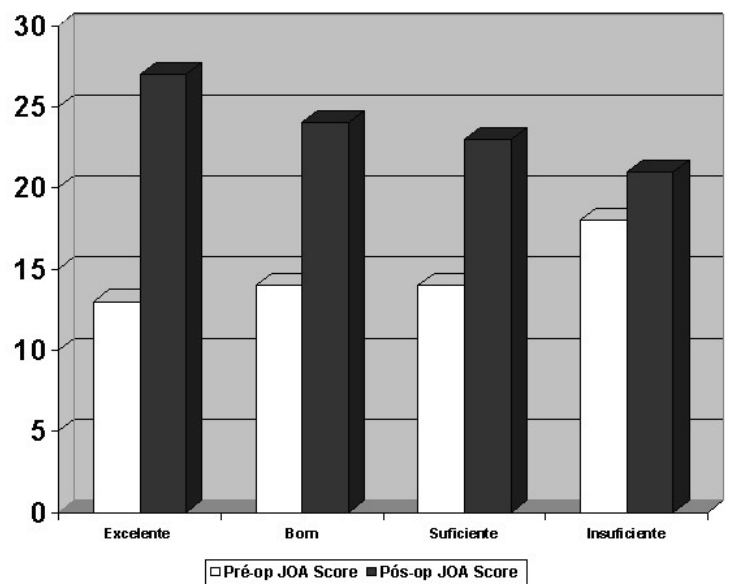

Figura 2

Comparação entre os valores pré e pós-operatório JOA em relação aos resultados de melhoria clínica (IR). $51 \%$ da população teve excelente (IR $\geq 80 \%), 24 \%$ bom (IR=65-80\%), $16 \%$ suficiente $(I R=50-65 \%)$ e $9 \%$ insuficiente $(I R<50 \%)$. 
inicial e o atraso para a cirurgia (dados não publicados). Piores resultados foram alcançados quando o atraso para a cirurgia foi superior a um ano. Contudo, a cirurgia precoce (0-6 meses) também não aumentou significativamente o resultado. Estes dados sugerem que a indicação cronológica ideal para a cirurgia não é previsível por esta abordagem.
Dos 68 entrevistados, 8 foram reoperados durante o período de seguimento. Destes, 7 foram devido a recorrências da hérnia discal no mesmo nível, enquanto um caso foi devido a um erro inicial na identificação do espaço discal afectado. Um doente teve uma infecção tecidular superficial, resolvida com drenagem e antibioterapia dirigida. Não houve quaisquer outras complicações cirúrgicas

\section{TABELA 2 - The Japanese Orthopaedic Association Scores for Assessment of Low Back Pain}

\begin{tabular}{|c|c|c|c|}
\hline \multicolumn{3}{|c|}{ Categories } & Score \\
\hline \multicolumn{4}{|l|}{ I. Subjective symptoms (9 points) } \\
\hline \multicolumn{4}{|l|}{ A. Low back pain } \\
\hline \multicolumn{3}{|l|}{ a. None } & 3 \\
\hline \multicolumn{3}{|c|}{ b. Occasional mild pain } & 2 \\
\hline \multicolumn{3}{|c|}{ c. Frequent mild or occasional severe pain } & 1 \\
\hline \multicolumn{3}{|l|}{ d. Frequent or continuous severe pain } & 0 \\
\hline \multicolumn{4}{|l|}{ B. Leg pain and/or tingling } \\
\hline \multicolumn{3}{|l|}{ a. None } & 3 \\
\hline \multicolumn{3}{|l|}{ b. Occasional slight symptoms } & 2 \\
\hline \multicolumn{3}{|c|}{ c. Frequent slight or occasional severe symptoms } & 1 \\
\hline \multicolumn{3}{|c|}{ d. Frequent or continuous severe symptoms } & 0 \\
\hline \multicolumn{4}{|c|}{ C. Gait } \\
\hline \multicolumn{3}{|l|}{ a. Normal } & 3 \\
\hline \multicolumn{3}{|c|}{ b. Able to walk further than $500 \mathrm{~m}$, although it results in pain, tingling, and/or muscle weakness } & 2 \\
\hline \multicolumn{3}{|c|}{ c. Unable to walk further than $500 \mathrm{~m}$ because of leg pain and/or muscle weakness } & 1 \\
\hline \multicolumn{3}{|c|}{ d. Unable to walk further than $100 \mathrm{~m}$ because of leg pain and/or muscle weakness } & 0 \\
\hline \multicolumn{4}{|c|}{ II. Clinical signs (6 points) } \\
\hline \multicolumn{4}{|c|}{ A. Straight-leg raising test (including tight hamstrings) } \\
\hline \multicolumn{3}{|l|}{ a. Normal } & 2 \\
\hline \multicolumn{3}{|l|}{ b. $30^{\circ}-70^{\circ}$} & 1 \\
\hline \multicolumn{3}{|l|}{ c. $<30^{\circ}$} & 0 \\
\hline \multicolumn{4}{|l|}{ B. Sensory disturbance } \\
\hline \multicolumn{3}{|l|}{ a. None } & 2 \\
\hline \multicolumn{3}{|l|}{ b. Slight disturbance (not subjective) } & 1 \\
\hline \multicolumn{3}{|l|}{ c. Marked disturbance } & 0 \\
\hline C. Motor disturbance (MNT) & & & \\
\hline a. Normal (Grade 5) & & & 2 \\
\hline b. Slight weakness (Grade 4) & & & 1 \\
\hline c. Marked weakness (Grade 3-0) & & & 0 \\
\hline & Severe & Moderate & None \\
\hline III. Restriction of ADL (14 points) & & & \\
\hline Turning over while lying & 0 & 1 & 2 \\
\hline Standing & 0 & 1 & 2 \\
\hline Washing & 0 & 1 & 2 \\
\hline Leaning forward & 0 & 1 & 2 \\
\hline Sitting $(1 \mathrm{hr})$ & 0 & 1 & 2 \\
\hline Liffing or holding & 0 & 1 & 2 \\
\hline Walking & 0 & 1 & 2 \\
\hline IV. Urinary function (-6 points) & & & \\
\hline a. Normal & & 0 & \\
\hline b. Mild dysuria & & -3 & \\
\hline c. Severe dysuria & & -6 & \\
\hline
\end{tabular}

The completely normal condition is a total of the best scores $(I+I I+I I I+I V)=29$ points.

Versão em inglês adaptada ${ }^{23}$ 
descritas, tais como lesões durais ou da raiz, síndrome da cauda equina ou espondilólise ${ }^{2}$. A comparação dos resultados bom e excelente dos subgrupos ao resultado insuficiente revelou uma taxa de reintervenção cirúrgica significativamente superior $(\mathrm{p}<0,05)$ no último subgrupo (cerca de 50\%) (Tabela 1). Embora a taxa de complicações seja universalmente superior nos grupos com piores resultados, é pertinente realçar que 5 dos 7 casos de recorrência e a infecção superficial ocorreram nos subgrupos com resultados excelente e bom. Destes últimos pacientes, apenas um não voltou à actividade laboral e outro teve de mudar para um trabalho com funções mais leves.

Como era de se esperar, o retorno à prévia actividade laboral foi significativamente superior entre pacientes com resultados bom e excelente, em comparação à reduzida capacidade de trabalho (25\%), daqueles que obtiveram resultados piores. Oito dos pacientes acompanhados não regressaram ao trabalho; três deles, com idade superior a 50 anos, reformaram-se, um apresentava concomitantemente uma coxartrose, e outro continuava desempregado no final do estudo. A necessidade de mudar de emprego por incapacidade de execução das funções que exerciam previamente ocorreu com cinco pacientes.

$\mathrm{O}$ escore JOA pré-operatório aumentou gradualmente (Figura 3) para cada parâmetro avaliado nos pacientess com resultados bom e excelente, sugerindo que a debilidade inicial alta (score inicial baixo) está positivamente correlacionada com melhores resultados ao final do seguimento. Particularmente, em relação aos sintomas subjectivos (lombalgia, radiculalgia e incapacidade de marcha), estes mostraram valores mínimos nos grupos com melhores resultados em comparação com o grupo com piores
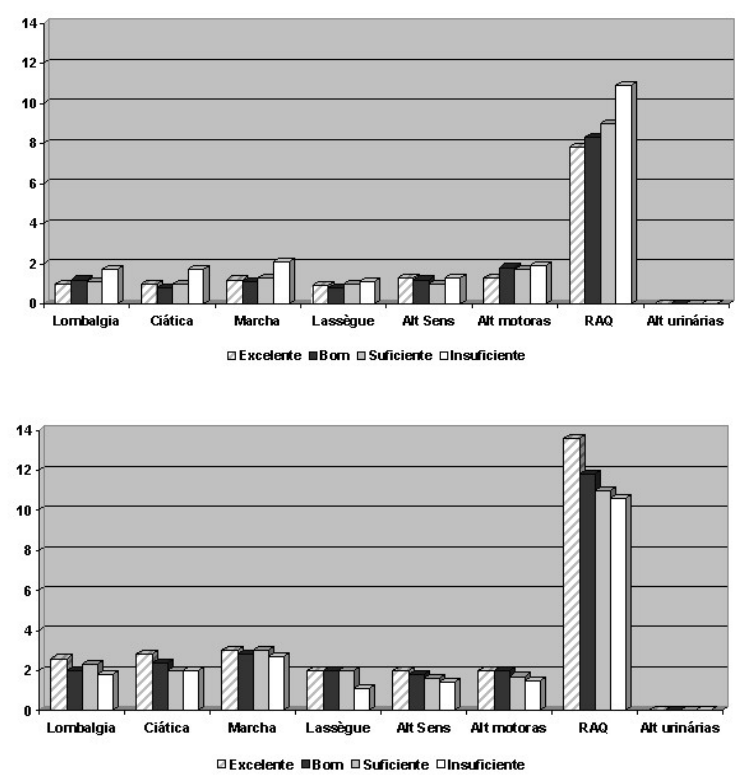

Figura 3

Comparação dos valores JOA pré (A) e pós-operatório (B), por categorias, de acordo com o resultado final. resultados, indicando que o escore JOA pré-operatório para sintomas subjectivos correlaciona-se inversamente com o resultado da discectomia lombar. Os resultados levantaram questões acerca da necessidade de cirurgia imediata quando a soma do escore JOA pré-operatório para os sinais clínicos (teste de Lassègue, alterações sensitivas ou motoras) for superior a 3 (isto é, debilidade objectiva inicial baixa), uma vez que, nesta situação, foram obtidos resultados bom e excelente mesmo quando a cirurgia teve um atraso superior a um ano.

Pela análise multifatorial, as restrições às actividades da vida quotidiana e a preponderância dos sintomas subjectivos sobre os sinais clínicos, foram associados com melhores resultados clínicos.

\section{DISCUSSÃO}

Em contraste com os resultados de várias publicações sugestivos de que a cirurgia de discectomia lombar é essencialmente paliativa ${ }^{10}$, a série apresentada demonstra que a microdiscectomia lombar selectiva em pacientes com hérnia discal lombar sem melhoria clínica após 8 semanas de tratamento conservador conduziu a resultados bom e excelente em mais de $75 \%$ dos casos. Estes resultados apresentam taxas de sucesso semelhantes às publicadas por outros autores $^{15,16}$. Os critérios de selecção dos doentes parecem ser determinantes para a obtenção de bons resultados finais. Os critérios de exclusão, na nossa série, incluíram a existência de patologias associadas da coluna lombar, doenças sistémicas, fumadores e toxicodependentes, uma vez que tais factores podem influenciar clinicamente o resultado da discectomia ${ }^{6,12}$. A idade média dos doentes foi de 40 anos, não influenciando, aparentemente, o prognóstico da discectomia lombar, uma vez que uma idade semelhante foi homogeneamente encontrada entre todos os subgrupos. Similarmente a outras publicações ${ }^{17}$ o género feminino esteve associado a pior prognóstico.

A utilização da cirurgia como forma de tratamento para hérnia discal lombar varia enormemente entre países. Acredita-se que a razão principal de insucesso seja a má selecção de candidatos à cirurgia. Na literatura, discute-se frequentemente a necessidade de avaliação psicossocial, na tentativa de prever um resultado bom ou ruim, especialmente em pacientes sem indicação formal para a cirurgia.

Doentes com queixas clínicas moderadas, principalmente patologias psíquicas (p. e., hipocondria, histeria, paranoia e esquizofrenia) ${ }^{18}$, que também têm problemas laborais ou estão a receber subsídios ${ }^{17}$, apresentam maiores dificuldades de lidar com a patologia e revelam pior satisfação pós-operatória. Estes trabalhos indicam que o uso de sistemas de classificação objectivos reduz a incidência de achados negativos aquando da cirurgia, assim como melhores resultados clínicos após discectomia selectiva. Todos os doentes incluídos na nossa série apresentavam hérnia discal lombar confirmada por tomografia computorizada ou ressonância magnética. A maior parte $(85 \%)$ teve radiculalgia com défice neurológico associado durante 
um mínimo de 6 semanas, que se apresentam indicações formais para cirurgia, por outro lado evitando proposição para cirurgia de pacientes com queixas psicossociais não relacionadas a hérnias discais lombares.

Quanto mais elevado o escore JOA pré-operatório (>15), maior a probabilidade de o paciente obter um pior resultado. A eficácia da previsão dos resultados ruins é significativamente aumentada se o somatório do escore JOA pré-operatório, para sinais objectivos, for superior a 3. Este facto deve questionar a necessidade de cirurgia imediata em doentes com queixas neurológicas escassas. Embora a maioria dos doentes tenham ocasionalmente tido, após a cirurgia, lombalgia ou radiculalgia, a descrição da sua capacidade de marcha foi praticamente normal, e a maioria tolerou bem estes sintomas de intensidade moderada. Outro ponto de interesse reflecte-se na capacidade de realização de actividades da vida quotidiana, que também podem estar relacionadas com fenómenos psicossociais. Paralelamente aos nossos achados, a avaliação prévia de questionários subjectivos mostrou que uma debilidade inicial baixa correlaciona-se negativamente com o resultado final ${ }^{17,19}$.

A nossa série demonstra também claramente que o atraso da cirurgia superior a um ano correlaciona-se com piores resultados. Contudo, a cirurgia precoce (0-6 meses) também não melhorou o prognóstico dado, tendo sido encontrada uma distribuição percentual semelhante entre todos os grupos quando comparados à população total. Alguns autores sugerem melhores resultados nos pacientes operados imediatamente após indicação formal ${ }^{20,21}$. O atraso da cirurgia pode agravar problemas laborais ou de subsídios, o que, em última análise, aumentará a insatisfação. É de realçar que, nesta série, 20 dos 23 pacientes cuja cirurgia sofreu um atraso superior a 6 meses tenham obtido excelentes ou bons resultados clínicos. A percentagem de pacientes que regressaram ao trabalho anterior foi notavelmente relacionada com a taxa de melhoria percentual calculada a partir do ecore clínico JOA pré e pós-operatório, reforçando o valor objectivo deste sistema de avaliação na previsão da posterior capacidade laboral.

A taxa de nova intervenção $(11,7 \%)$ foi semelhante à encontrada noutras publicações ${ }^{22,23}$. Embora a percentagem de complicações fosse inversamente correlacionada com a melhoria da situação (Tabela 1), os resultados indicam igualmente que a sua frequência não justifica piores resultados, uma vez que 5 das 7 recidivas, assim como a infecção superficial, ocorreram nos grupos com resultados bons e excelentes. Estes dados apontam que outras variáveis, para além das complicações, devem ser consideradas para justificar resultados pobres das discectomias lombares. Adicionalmente, estes achados sugerem que as recidivas não afectam o prognóstico a longo prazo, embora seja necessária uma nova intervenção cirúrgica.

\section{CONCLUSÃO}

Os resultados desta análise multifatorial sugerem que o uso clínico do escore JOA é facilmente exequível a clínicos e pacientes. Permite uniformizar dados subjectivos e apresenta elevado valor preditivo dos resultados da discectomia lombar.

\section{REFERÊNCIAS}

1. Frymoyer JW. Back pain and sciatica. N Engl J Med. 1988;318(5):291-300. Review.

2. Bigos SJ, Bowyer O, Braen GR, BrownK, Deyo R, Haldeman S, et al. Acute Low Back Problems in Adults: Clinical Practice Guideline No. 14. AHCPR Publication No. 95-0642. Rockville, MD: Agency for Health Care Policy and Research, Public Health Service, U.S. Department of Health and Human Services. December 1994. [Internet] [cited 2010 Apr 20]. Available from: http://www. ncbi.nlm.nih.gov/bookshelf/br.fcgi?bo $\mathrm{ok}=\mathrm{hsarchive} \&$ part $=\mathrm{A} 25870$

3. Hakelius A. Prognosis in sciatica. A clinical follow-up of surgical and nonsurgical treatment. Acta Orthop Scand Suppl. 1970;129:1-76.

4. Barrios C, Ahmed M, Arrotegui JI, Björnsson A. Clinical factors predicting outcome after surgery for herniated lumbar disc: an epidemiological multivariate analysis. J. Spinal Disord. 1990;3(3):205-9.
5. Boden SD, Davis DO, Dina TS, Patronas NJ, Wiesel SW. Abnormal magnetic-resonance scans of the lumbar spine in asymptomatic subjects. A prospective investigation. J. Bone Joint Surg Am. 1990;72(3):403-8.

6. Woertgen C, Holzschuh M, Rothoerl RD, Brawanski A. Does the choice of outcome scale influence prognostic factors for lumbar disc surgery? A prospective, consecutive study of 121 patients. Eur Spine J. 1997;6(3):17380.

7. Falck B, Nykvist F, Hurme M, Alarante H. Prognostic value of EMG in patients with lumbar disc herniation - a five year follow up. Electromyogr Clin Neurophysiol. 1993;33(1):19-26.

8. Deyo RA, Cherkin DC, Loeser JD, Bigos SJ, Ciol MA. Morbidity and mortality in association with operations on the lumbar spine. The influence of age, diagnosis, and procedure. J. Bone Joint Surg Am. 1992;74(4):536-43.
9. Larequi-Lauber T, Vader JP, Burnand B, Brook RH, Kosecoff J, Sloutskis D, et al. Appropriateness of indications for surgery of lumbar disc hernia and spinal stenosis. Spine (Phila Pa 1976). 1997;22(2):203-9.

10. Weber H. Lumbar disc herniation. A controlled, prospective study with ten years of observation. Spine (Phila Pa 1976). 1983;8(2):131-40.

11.Spengler DM, Ouellette EA, Battié M, Zeh J. Elective discectomy for herniation of a lumbar disc. Additional experience with an objective method. J Bone Joint Surg Am. 1990;72(2):2307.

12. Waddell G, Main CJ, Morris EW, Di Paola MP, Gray IC. Chronic low-back pain, psychologic distress, and illness behavior. Spine (Phila Pa 1976). 1984;9(2):209-13.

13.Kulali A, von Wild K. Lumbar spinal surgery for sciatica due to intervertebral disc disease in the elderly. Neurosurg Rev. 1996;19(3):157-62. 
14.Korres DS, Loupassis G, Stamos K. Results of lumbar discectomy: a study using 15 different evaluation methods. Eur Spine J. 1992;1(1):20-4.

15.Nygaard OP, Kloster R, Mellgreen SI. Recovery of sensory nerve fibres after surgical decompression in lumbar radiculopathy: use of quantitative sensory testing in the exploration of different populations of nerve fibres. J Neurol Neurosurg Psychiatry 1998;64(1):120-3.

16. Herron LD, Turner JA, Novell LA, Kreif SL. Patient selection for lumbar discectomy with a revised objective rating system. Clin Orthop Relat Res. 1996;(325):148-55.

17.Nygaard OP, Romner B, Trumpy JH. Duration of symptoms as a predictor of outcome after lumbar disc surgery. Acta Neurochir (Wien). 1994;128(14):53-6.
18.Herron LD, Turner JA, Weiner P. Lumbar disc herniations: the predictive value of the Health Attribution Test (HAT) and the Minnesota Multiphasic Personality Inventory (MMPI). J Spinal Disord. 1988;1(1):2-8.

19.Hurme M, Alaranta H. Factors predicting the result of surgery for lumbar intervertebral disc herniation. Spine (Phila Pa 1976). 1987;12(9):933-8.

20.Jönsson B, Strömqvist B. The straight leg raising test and the severity of symptoms in lumbar disc herniation. A preoperative evaluation. Spine (Phila Pa 1976). 1995;20(1):27-30.

21.Simpson JM, Silveri CP, Balderston RA, Simeone FA, An HS. The results of operations on the lumbar spine in patients who have diabetes mellitus. J Bone Join Surg Am. 1993;75(12):1823-9.
22.Pappas CT, Harrington T, Sonntag VK. Outcome analysis in 654 surgically treated lumbar disc herniations. Neurosurgery. 1992;30(6):862-6.

23.Japanese Orthopaedic Association. Assessment of surgical treatment of low back pain. J Jpn Orthop Assoc. 1984;58:1183-6.

\section{Correspondência}

Paulo Jorge da Rocha Carvalho Serviço de Ortopedia e Traumatologia Centro Hospitalar de Vila Nova de Gaia Rua Dr. Francisco Sá Carneiro 4400-129 Vila Nova de Gaia - Portugal E-mail:pircarvalho@gmail.com 